\title{
Measurement of the $\boldsymbol{B} \rightarrow \boldsymbol{X}_{s} \ell^{+} \ell^{-}$Branching Fraction and Search for Direct $\boldsymbol{C P}$ Violation from a Sum of Exclusive Final States
}

J. P. Lees, ${ }^{1}$ V. Poireau, ${ }^{1}$ V. Tisserand, ${ }^{1}$ E. Grauges, ${ }^{2}$ A. Palano, ${ }^{3 a, 3 b}$ G. Eigen, ${ }^{4}$ B. Stugu, ${ }^{4}$ D. N. Brown,${ }^{5}$ L. T. Kerth, ${ }^{5}$ Yu. G. Kolomensky, ${ }^{5}$ M. J. Lee, ${ }^{5}$ G. Lynch, ${ }^{5}$ H. Koch, ${ }^{6}$ T. Schroeder, ${ }^{6}$ C. Hearty, ${ }^{7}$ T. S. Mattison, ${ }^{7}$ J. A. McKenna, ${ }^{7}$ R. Y. So, ${ }^{7}$ A. Khan, ${ }^{8}$ V. E. Blinov, ${ }^{9 a, 9 c}$ A. R. Buzykaev, ${ }^{9 a}$ V. P. Druzhinin, ${ }^{9 a, 9 b}$ V. B. Golubev, ${ }^{9 a, 9 b}$ E. A. Kravchenko, ${ }^{9 a, 9 b}$

A. P. Onuchin, ${ }^{9 a, 9 c}$ S. I. Serednyakov, ${ }^{9 a}, 9 b$ Yu. I. Skovpen, ${ }^{9 a, 9 b}$ E. P. Solodov, ${ }^{9 a, 9 b}$ K. Yu. Todyshev, ${ }^{9 a, 9 b}$ A. N. Yushkov, ${ }^{9 a}$ A. J. Lankford, ${ }^{10}$ M. Mandelkern, ${ }^{10}$ B. Dey, ${ }_{11}$ J. W. Gary, ${ }^{11}$ O. Long, ${ }^{11}$ C. Campagnari, ${ }^{12}$ M. Franco Sevilla, ${ }^{12}$ T. M. Hong, ${ }^{12}$ D. Kovalskyi, ${ }^{12}$ J. D. Richman, ${ }^{12}$ C. A. West, ${ }^{12}$ A. M. Eisner, ${ }^{13}$ W. S. Lockman, ${ }^{13}$ B. A. Schumm, ${ }^{13}$ A. Seiden, ${ }^{13}$ D. S. Chao, ${ }^{14}$ C. H. Cheng, ${ }^{14}$ B. Echenard, ${ }^{14}$ K. T. Flood, ${ }^{14}$ D. G. Hitlin, ${ }^{14}$ T. S. Miyashita, ${ }^{14}$ P. Ongmongkolkul, ${ }^{14}$ F. C. Porter,${ }^{14}$ R. Andreassen, ${ }^{15}$ Z. Huard, ${ }^{15}$ B. T. Meadows, ${ }^{15}$ B. G. Pushpawela, ${ }^{15}$ M. D. Sokoloff, ${ }^{15}$ L. Sun, ${ }^{15}$ P. C. Bloom, ${ }^{16}$ W. T. Ford ${ }^{16}$ A. Gaz ${ }^{16}$ U. Nauenberg, ${ }^{16}$ J. G. Smith ${ }^{16}$ S. R. Wagner,${ }^{16}$ R. Ayad, ${ }^{17, \dagger}$ W. H. Toki, ${ }^{17}$ B. Spaan, ${ }^{18}$ R. Schwierz, ${ }^{19}$ D. Bernard ${ }^{20}$ M. Verderi, ${ }^{20}$ S. Playfer, ${ }^{21}$ D. Bettoni, ${ }^{22 a}$ C. Bozzi, ${ }^{22 a}$ R. Calabrese, ${ }^{22 a, 22 b}$ G. Cibinetto, ${ }^{22 a, 22 b}$ E. Fioravanti, ${ }^{22 a, 22 b}$ I. Garzia, ${ }^{22 a, 22 b}$ E. Luppi, ${ }^{22 a, 22 b}$ L. Piemontese, ${ }^{22 a}$ V. Santoro, ${ }^{22 a}$ A. Calcaterra, ${ }^{23}$ R. de Sangro, ${ }^{23}$ G. Finocchiaro, ${ }^{23}$ S. Martellotti, ${ }^{23}$ P. Patteri, ${ }^{23}$ I. M. Peruzzi, ${ }^{23,}$ M. Piccolo, ${ }^{23}$ M. Rama, ${ }^{23}$ A. Zallo, ${ }^{23}$ R. Contri, ${ }^{24 a, 24 b}$ E. Guido,${ }^{24 a, 24 b}$ M. Lo Vetere, ${ }^{24 a, 24 b}$ M. R. Monge, ${ }^{24 a, 24 b}$ S. Passaggio, ${ }^{24 a}$ C. Patrignani, ${ }^{24 a, 24 b}$ E. Robutti, ${ }^{24 a}$ B. Bhuyan, ${ }^{25}$ V. Prasad, ${ }^{25}$ M. Morii, ${ }^{26}$ A. Adametz, ${ }^{27}$ U. Uwer, ${ }^{27}$ H. M. Lacker, ${ }^{28}$ P. D. Dauncey, ${ }^{29}$ U. Mallik,${ }^{30}$ C. Chen, ${ }^{31}$ J. Cochran, ${ }^{31}$ W. T. Meyer, ${ }^{31}$ S. Prell, ${ }^{31}$ H. Ahmed, ${ }^{32}$ A. V. Gritsan, ${ }^{33}$ N. Arnaud,${ }^{34}$ M. Davier, ${ }^{34}$ D. Derkach,${ }^{34}$ G. Grosdidier,${ }^{34}$ F. Le Diberder, ${ }^{34}$ A. M. Lutz, ${ }^{34}$ B. Malaescu, ${ }^{34,8}$ P. Roudeau, ${ }^{34}$ A. Stocchi,${ }^{34}$ G. Wormser, ${ }^{34}$ D. J. Lange,${ }^{35}$ D. M. Wright, ${ }^{35}$ J. P. Coleman, ${ }^{36}$ J. R. Fry ${ }^{36}$ E. Gabathuler, ${ }^{36}$ D. E. Hutchcroft, ${ }^{36}$ D. J. Payne, ${ }^{36}$ C. Touramanis, ${ }^{36}$ A. J. Bevan, ${ }^{37}$ F. Di Lodovico, ${ }^{37}$ R. Sacco, ${ }^{37}$ G. Cowan, ${ }^{38}$ J. Bougher, ${ }^{39}$ D. N. Brown, ${ }^{39}$ C. L. Davis, ${ }^{39}$ A. G. Denig, ${ }^{40}$ M. Fritsch, ${ }^{40}$ W. Gradl, ${ }^{40}$ K. Griessinger, ${ }^{40}$ A. Hafner, ${ }^{40}$ E. Prencipe, ${ }^{40}$ K. R. Schubert, ${ }^{40}$ R. J. Barlow, ${ }^{41, \|}$ G. D. Lafferty, ${ }^{41}$ R. Cenci, ${ }^{42}$ B. Hamilton, ${ }^{42}$ A. Jawahery, ${ }^{42}$ D. A. Roberts, ${ }^{42}$ R. Cowan, ${ }^{43}$ D. Dujmic, ${ }^{43}$ G. Sciolla, ${ }^{43}$ R. Cheaib, ${ }^{44}$ P. M. Patel,,${ }^{44, *}$ S. H. Robertson, ${ }^{44}$ P. Biassoni, ${ }^{45 a, 45 b}$ N. Neri, ${ }^{45 a}$ F. Palombo, ${ }^{45 a, 45 b}$ L. Cremaldi, ${ }^{46}$ R. Godang, ${ }^{46,}$ P. Sonnek, ${ }^{46}$ D. J. Summers, ${ }^{46}$ M. Simard, ${ }^{47}$ P. Taras,${ }^{47}$ G. De Nardo,${ }^{48 a, 48 b}$ D. Monorchio, ${ }^{48 a, 48 b}$ G. Onorato, ${ }^{48 a, 48 b}$ C. Sciacca, ${ }^{48 a, 48 b}$ M. Martinelli, ${ }^{49}$ G. Raven,${ }^{49}$ C. P. Jessop, ${ }^{50}$ J. M. LoSecco,${ }^{50}$ K. Honscheid ${ }^{51}$ R. Kass,${ }^{51}$ J. Brau, ${ }^{52}$ R. Frey, ${ }^{52}$ N. B. Sinev, ${ }^{52}$ D. Strom,${ }^{52}$ E. Torrence, ${ }^{52}$ E. Feltresi,${ }^{53 a, 53 b}$ M. Margoni,${ }^{53 a, 53 b}$ M. Morandin, ${ }^{53 a}$ M. Posocco, ${ }^{53 a}$ M. Rotondo, ${ }^{53 a}$ G. Simi, ${ }^{53 a, 53 b}$ F. Simonetto, ${ }^{53 a, 53 b}$ R. Stroili, ${ }^{53 a, 53 b}$ S. Akar, ${ }^{54}$ E. Ben-Haim, ${ }^{54}$ M. Bomben, ${ }^{54}$ G. R. Bonneaud,${ }^{54}$ H. Briand, ${ }^{54}$ G. Calderini, ${ }^{54}$ J. Chauveau, ${ }^{54} \mathrm{Ph}$. Leruste, ${ }^{54} \mathrm{G}$. Marchiori, ${ }^{54} \mathrm{~J}$. Ocariz, ${ }^{54} \mathrm{~S}$. Sitt, ${ }^{54}$ M. Biasini,${ }^{55 a, 55 b}$ E. Manoni, ${ }^{55 \mathrm{a}}$

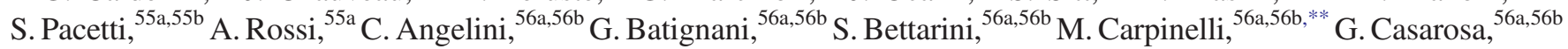
A. Cervelli, ${ }^{56 a, 56 b}$ M. Chrzaszcz, ${ }^{56 a, 56 b}$ F. Forti, ${ }^{56 a, 56 b}$ M. A. Giorgi, ${ }^{56 a, 56 b}$ A. Lusiani, ${ }^{56 a, 56 c}$ B. Oberhof, ${ }^{56 a, 56 b}$ E. Paoloni, ${ }^{56 a, 56 b}$ A. Perez, ${ }^{56 \mathrm{a}}$ G. Rizzo, ${ }^{56 a, 56 \mathrm{~b}}$ J. J. Walsh ${ }^{56 \mathrm{a}}$ D. Lopes Pegna, ${ }^{57}$ J. Olsen,${ }^{57}$ A. J. S. Smith, ${ }^{57}$ R. Faccini, ${ }^{58,58 \mathrm{~b}}$ F. Ferrarotto, ${ }^{58 \mathrm{a}}$ F. Ferroni, ${ }^{58 a, 58 b}$ M. Gaspero ${ }^{58 a, 58 b}$ L. Li Gioi, ${ }^{58 \mathrm{a}}$ G. Piredda, ${ }^{58 \mathrm{a}}$ C. Bünger, ${ }^{59}$ O. Grünberg, ${ }^{59}$ T. Hartmann,${ }^{59}$ T. Leddig, ${ }^{59}$ C. Voß ${ }^{59}$ R. Waldi, ${ }^{59}$ T. Adye, ${ }^{60}$ E. O. Olaiya,${ }^{60}$ F. F. Wilson, ${ }^{60}$ S. Emery, ${ }^{61}$ G. Hamel de Monchenault,${ }^{61}$ G. Vasseur, ${ }^{61}$

Ch. Yèche, ${ }^{61}$ F. Anulli, ${ }^{62, \dagger \dagger}$ D. Aston, ${ }^{62}$ D. J. Bard ${ }^{62}$ J. F. Benitez, ${ }^{62}$ C. Cartaro, ${ }^{62}$ M. R. Convery, ${ }^{62}$ J. Dorfan, ${ }^{62}$

G. P. Dubois-Felsmann, ${ }^{62}$ W. Dunwoodie, ${ }^{62}$ M. Ebert, ${ }^{62}$ R. C. Field, ${ }^{62}$ B. G. Fulsom, ${ }^{62}$ A. M. Gabareen, ${ }^{62}$ M. T. Graham, ${ }^{62}$ C. Hast, ${ }^{62}$ W. R. Innes, ${ }^{62}$ P. Kim, ${ }^{62}$ M. L. Kocian, ${ }^{62}$ D. W. G. S. Leith,${ }^{62}$ P. Lewis, ${ }^{62}$ D. Lindemann, ${ }^{62}$ B. Lindquist, ${ }^{62}$ S. Luitz, ${ }^{62}$ V. Luth, ${ }^{62}$ H. L. Lynch, ${ }^{62}$ D. B. MacFarlane, ${ }^{62}$ D. R. Muller, ${ }^{62}$ H. Neal,${ }^{62}$ S. Nelson, ${ }^{62}$ M. Perl, ${ }^{62}$ T. Pulliam, ${ }^{62}$ B. N. Ratcliff, ${ }^{62}$ A. Roodman, ${ }^{62}$ A. A. Salnikov ${ }^{62}$ R. H. Schindler, ${ }^{62}$ A. Snyder ${ }^{62}$ D. Su, ${ }^{62}$ M. K. Sullivan, ${ }^{62}$ J. Va'vra, ${ }^{62}$ A. P. Wagner, ${ }^{62}$ W. F. Wang, ${ }^{62}$ W. J. Wisniewski, ${ }^{62}$ M. Wittgen, ${ }^{62}$ D. H. Wright,${ }^{62}$ H. W. Wulsin, ${ }^{62}$ V. Ziegler, ${ }^{62}$ W. Park ${ }^{63}$ M. V. Purohit, ${ }^{63}$ R. M. White, ${ }^{63, *}$ J. R. Wilson, ${ }^{63}$ A. Randle-Conde, ${ }^{63}$ S. J. Sekula, ${ }^{64}$ M. Bellis, ${ }^{65}$ P. R. Burchat, ${ }^{65}$ E. M. T. Puccio, ${ }^{65}$ M. S. Alam, ${ }^{66}$ J. A. Ernst, ${ }^{66}$ R. Gorodeisky, ${ }^{67}$ N. Guttman, ${ }^{67}$ D. R. Peimer, ${ }^{67}$ A. Soffer, ${ }^{67}$ S. M. Spanier, ${ }^{68}$ J. L. Ritchie, ${ }^{69}$ A. M. Ruland, ${ }^{69}$ R. F. Schwitters, ${ }^{69}$ B. C. Wray, ${ }^{69}$ J. M. Izen, ${ }^{70}$ X. C. Lou ${ }^{70}$ F. Bianchi, ${ }^{71 a, 71 b}$

F. De Mori, ${ }^{71 a, 71 b}$ A. Filippi, ${ }^{71 a}$ D. Gamba, ${ }^{71 a, 71 b}$ S. Zambito, ${ }^{71 a, 71 b}$ L. Lanceri, ${ }^{72 a, 72 b}$ L. Vitale, ${ }^{72 a, 72 b}$ F. Martinez-Vidal ${ }^{73}$ A. Oyanguren, ${ }^{73}$ P. Villanueva-Perez,${ }^{73}$ J. Albert, ${ }^{74}$ Sw. Banerjee, ${ }^{74}$ F. U. Bernlochner, ${ }^{74}$ H. H. F. Choi,${ }^{74}$ G. J. King, ${ }^{74}$

R. Kowalewski, ${ }^{74}$ M. J. Lewczuk, ${ }^{74}$ T. Lueck, ${ }^{74}$ I. M. Nugent, ${ }^{74}$ J. M. Roney, ${ }^{74}$ R. J. Sobie, ${ }^{74}$ N. Tasneem, ${ }^{74}$

T. J. Gershon, ${ }^{75}$ P. F. Harrison, ${ }^{75}$ T. E. Latham, ${ }^{75}$ H. R. Band, ${ }^{76}$ S. Dasu, ${ }^{76}$ Y. Pan, ${ }^{76}$ R. Prepost, ${ }^{76}$ and S. L. $\mathrm{Wu}^{76}$

(BABAR Collaboration) 
${ }^{1}$ Laboratoire d'Annecy-le-Vieux de Physique des Particules (LAPP), Université de Savoie, CNRS/IN2P3, F-74941 Annecy-Le-Vieux, France

${ }^{2}$ Universitat de Barcelona, Facultat de Fisica, Departament ECM, E-08028 Barcelona, Spain

${ }^{3 a}$ INFN Sezione di Bari, I-70126 Bari, Italy

${ }^{3 \mathrm{~b}}$ Dipartimento di Fisica, Università di Bari, I-70126 Bari, Italy

${ }^{4}$ University of Bergen, Institute of Physics, N-5007 Bergen, Norway

${ }^{5}$ Lawrence Berkeley National Laboratory and University of California, Berkeley, California 94720, USA

${ }^{6}$ Ruhr Universität Bochum, Institut für Experimentalphysik 1, D-44780 Bochum, Germany

${ }^{7}$ University of British Columbia, Vancouver, British Columbia, Canada V6 T 1Z1

${ }^{8}$ Brunel University, Uxbridge, Middlesex UB8 3PH, United Kingdom

${ }^{9 a}$ Budker Institute of Nuclear Physics SB RAS, Novosibirsk 630090

${ }^{9 \mathrm{~b}}$ Novosibirsk State University, Novosibirsk 630090

${ }^{9 \mathrm{c}}$ Novosibirsk State Technical University, Novosibirsk 630092, Russia

${ }^{10}$ University of California at Irvine, Irvine, California 92697, USA

${ }^{11}$ University of California at Riverside, Riverside, California 92521, USA

${ }^{12}$ University of California at Santa Barbara, Santa Barbara, California 93106, USA

${ }^{13}$ University of California at Santa Cruz, Institute for Particle Physics, Santa Cruz, California 95064, USA

${ }^{14}$ California Institute of Technology, Pasadena, California 91125, USA

${ }^{15}$ University of Cincinnati, Cincinnati, Ohio 45221, USA

${ }^{16}$ University of Colorado, Boulder, Colorado 80309, USA

${ }^{17}$ Colorado State University, Fort Collins, Colorado 80523, USA

${ }^{18}$ Technische Universität Dortmund, Fakultät Physik, D-44221 Dortmund, Germany

${ }^{19}$ Technische Universität Dresden, Institut für Kern- und Teilchenphysik, D-01062 Dresden, Germany

${ }^{20}$ Laboratoire Leprince-Ringuet, Ecole Polytechnique, CNRS/IN2P3, F-91128 Palaiseau, France

${ }^{21}$ University of Edinburgh, Edinburgh EH9 3JZ, United Kingdom

${ }^{22 a}$ INFN Sezione di Ferrara, I-44122 Ferrara, Italy

${ }^{22 \mathrm{~b}}$ Dipartimento di Fisica e Scienze della Terra, Università di Ferrara, I-44122 Ferrara, Italy

${ }^{23}$ INFN Laboratori Nazionali di Frascati, I-00044 Frascati, Italy

${ }^{24 a}$ INFN Sezione di Genova, I-16146 Genova, Italy

${ }^{24 \mathrm{~b}}$ Dipartimento di Fisica, Università di Genova, I-16146 Genova, Italy

${ }^{25}$ Indian Institute of Technology Guwahati, Guwahati, Assam, 781 039, India

${ }^{26}$ Harvard University, Cambridge, Massachusetts 02138, USA

${ }^{27}$ Universität Heidelberg, Physikalisches Institut, D-69120 Heidelberg, Germany

${ }^{28}$ Humboldt-Universität zu Berlin, Institut für Physik, D-12489 Berlin, Germany

${ }^{29}$ Imperial College London, London, SW7 2AZ, United Kingdom

${ }^{30}$ University of Iowa, Iowa City, Iowa 52242, USA

${ }^{31}$ Iowa State University, Ames, Iowa 50011-3160, USA

${ }^{32}$ Physics Department, Jazan University, Jazan 22822, Saudia Arabia

${ }^{33}$ Johns Hopkins University, Baltimore, Maryland 21218, USA

${ }^{34}$ Laboratoire de l'Accélérateur Linéaire, IN2P3/CNRS et Université Paris-Sud 11, Centre Scientifique d'Orsay, F-91898 Orsay Cedex, France

${ }^{35}$ Lawrence Livermore National Laboratory, Livermore, California 94550, USA

${ }^{36}$ University of Liverpool, Liverpool L69 7ZE, United Kingdom

${ }^{37}$ Queen Mary, University of London, London, E1 4NS, United Kingdom

${ }^{38}$ University of London, Royal Holloway and Bedford New College, Egham, Surrey TW20 OEX, United Kingdom

${ }^{39}$ University of Louisville, Louisville, Kentucky 40292, USA

${ }^{40}$ Johannes Gutenberg-Universität Mainz, Institut für Kernphysik, D-55099 Mainz, Germany

${ }^{41}$ University of Manchester, Manchester M13 9PL, United Kingdom

${ }^{42}$ University of Maryland, College Park, Maryland 20742, USA

${ }^{43}$ Massachusetts Institute of Technology, Laboratory for Nuclear Science, Cambridge, Massachusetts 02139, USA

${ }^{44}$ McGill University, Montréal, Québec, Canada H3 A $2 T 8$

${ }^{45 a}$ INFN Sezione di Milano, I-20133 Milano, Italy

${ }^{45 \mathrm{~b}}$ Dipartimento di Fisica, Università di Milano, I-20133 Milano, Italy

${ }^{46}$ University of Mississippi, University, Mississippi 38677, USA

${ }^{47}$ Université de Montréal, Physique des Particules, Montréal, Québec, Canada H3 C 3J7

${ }^{48 a}$ INFN Sezione di Napoli, I-80126 Napoli, Italy, I-80126 Napoli, Italy

${ }^{48 \mathrm{~b}}$ Dipartimento di Scienze Fisiche, Università di Napoli Federico II, I-80126 Napoli, Italy

${ }^{49}$ NIKHEF, National Institute for Nuclear Physics and High Energy Physics, NL-1009 DB Amsterdam, Netherlands

${ }^{50}$ University of Notre Dame, Notre Dame, Indiana 46556, USA

${ }^{51}$ Ohio State University, Columbus, Ohio 43210, USA 


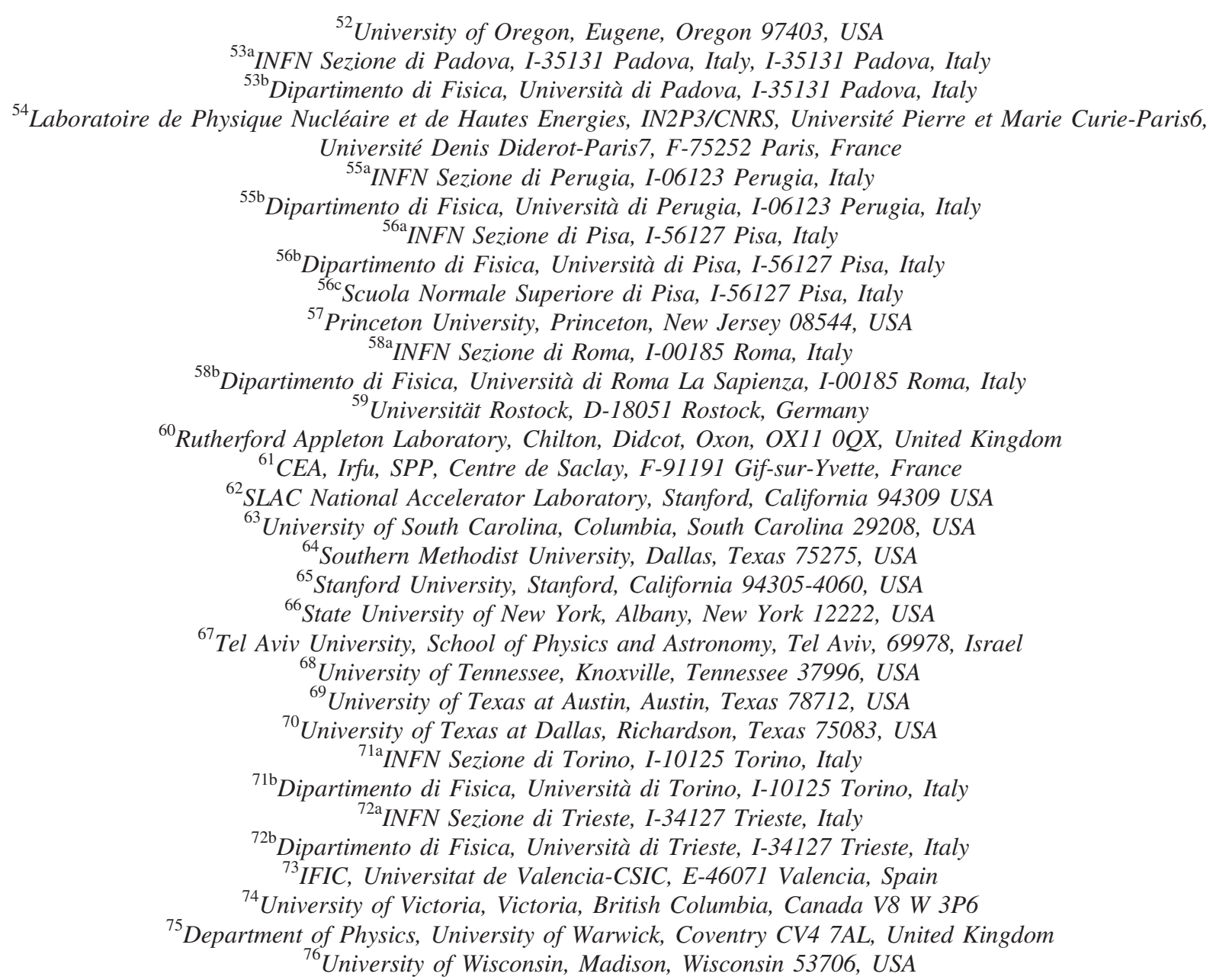

(Received 23 December 2013; revised manuscript received 21 April 2014; published 30 May 2014)

We measure the total branching fraction of the flavor-changing neutral-current process $B \rightarrow X_{s} \ell^{+} \ell^{-}$, along with partial branching fractions in bins of dilepton and hadronic system $\left(X_{s}\right)$ mass, using a sample of $471 \times 10^{6} \Upsilon(4 S) \rightarrow B \bar{B}$ events recorded with the $B A B A R$ detector. The admixture of charged and neutral $B$ mesons produced at PEP-II2 are reconstructed by combining a dilepton pair with 10 different $X_{s}$ final states. Extrapolating from a sum over these exclusive modes, we measure a lepton-flavor-averaged inclusive branching fraction $\mathcal{B}\left(B \rightarrow X_{s} \ell^{+} \ell^{-}\right)=\left[6.73_{-0.64}^{+0.70}(\text { stat })_{-0.25}^{+0.34}\right.$ (exp syst $) \pm 0.50($ model syst $\left.)\right] \times$ $10^{-6}$ for $m_{\ell^{+} \ell^{-}}^{2}>0.1 \mathrm{GeV}^{2} / c^{4}$. Restricting our analysis exclusively to final states from which a decaying $B$ meson's flavor can be inferred, we additionally report measurements of the direct $C P$ asymmetry $A_{C P}$ in bins of dilepton mass; over the full dilepton mass range, we find $A_{C P}=0.04 \pm 0.11 \pm 0.01$ for a leptonflavor-averaged sample.

DOI: 10.1103/PhysRevLett.112.211802

PACS numbers: 13.20.He, 11.30.Er

The $b \rightarrow s \ell^{+} \ell^{-}$transition, where $b$ is a bottom quark, $s$ is a strange quark, and $\ell^{+} \ell^{-}$is an $e^{+} e^{-}$or $\mu^{+} \mu^{-}$pair, is forbidden at lowest order in the standard model (SM) but is allowed at one loop via electroweak penguin and $W$-box diagrams. The amplitude for this decay is expressed in terms of perturbatively calculable effective Wilson coefficients, $C_{7}^{\text {eff }}, C_{9}^{\text {eff }}$, and $C_{10}^{\text {eff }}$, which represent the electromagnetic penguin diagram and the vector part and axial-vector part of the linear combination of the $Z$ penguin and $W^{+} W^{-}$box diagrams, respectively [1]. Non-SM contributions can enter these loops at the same order as the SM processes, modifying the Wilson coefficients from their SM expectations and allowing experimental sensitivity to possible non-SM physics [2-11].

We study the inclusive decay $B \rightarrow X_{s} \ell^{+} \ell^{-}$, where $X_{s}$ is a hadronic system containing exactly one kaon, using a sum over exclusive final states, which provides a basis for extrapolation to the fully inclusive rate. We measure the total branching fraction (BF), as well as partial BFs in five disjoint dilepton mass-squared $q^{2} \equiv m_{\ell^{+} \ell^{-}}^{2}$ bins and four 
hadronic mass $m_{X_{s}}$ bins, which are defined in Table I. We additionally search for direct $C P$ violation in the same $q^{2}$ bins. The relative precision of our results is approximately a factor of 2 better than the combined precision of all similar previously published measurements [12].

The $X_{s}$ system in the lowest mass $m_{X_{s}}$ bin $m_{X_{s}, 1}$ contains a single kaon with no other hadrons present; the $m_{X_{s}, 2}$ bin is populated only above the $K \pi$ threshold. Results are also reported in an additional $q^{2}$ region $q_{0}^{2} \equiv 1<q^{2}<6 \mathrm{GeV}^{2} / c^{4}$, i.e., the perturbative window away from the photon pole at low $q^{2}$ and the $c \bar{c}$ resonances at higher $q^{2}$, where theory uncertainties are well controlled [13-24]. The most recent SM predictions in this region are $\mathcal{B}^{\text {low }}\left(B \rightarrow X_{s} \mu^{+} \mu^{-}\right)=$ $(1.59 \pm 0.11) \times 10^{-6}$ and $\mathcal{B}^{\text {low }}\left(B \rightarrow X_{s} e^{+} e^{-}\right)=(1.64 \pm$ $0.11) \times 10^{-6}$ [22]. Theory uncertainties in the $q^{2}$ range above the $\psi(2 S)$ are also well characterized but relatively much larger than above, with SM predictions for $q^{2}>14.4 \mathrm{GeV}^{2} / c^{4}$ of $\mathcal{B}^{\text {high }}\left(B \rightarrow X_{s} \mu^{+} \mu^{-}\right)=(0.24 \pm$ $0.07) \times 10^{-6}$ and $\mathcal{B}^{\text {high }}\left(B \rightarrow X_{s} e^{+} e^{-}\right)=(0.21 \pm 0.07) \times$ $10^{-6}$ [22]. The SM expectation in the $q^{2}>4 m_{\mu}^{2}$ range is $\mathcal{B}\left(B \rightarrow X_{s} \ell^{+} \ell^{-}\right)=(4.6 \pm 0.8) \times 10^{-6}$ [20]. Direct $C P$ violation, defined as $A_{C P} \equiv\left(\mathrm{BF}_{\bar{b}}-\mathrm{BF}_{b}\right) /\left(\mathrm{BF}_{\bar{b}}+\mathrm{BF}_{b}\right)$, where $b(\bar{b})$ denotes a $\bar{B}(B)$ parent, is expected to be suppressed well below the $1 \%$ level in both exclusive and inclusive $b \rightarrow s \ell^{+} \ell^{-}$transitions [25-28]; however, in beyond-SM models with four quark generations, significant enhancements are possible, particularly in the high- $q^{2}$ region $[10,11]$.

The BABAR [29] and Belle [30] Collaborations have previously published $B \rightarrow X_{s} \ell^{+} \ell^{-}$BFs based on a sum over exclusive final states using only $\sim 25 \%$ of each experiment's final data set. More recently, both collaborations (along with
$\mathrm{LHCb}$ and $\mathrm{CDF}$ ) have published BFs, and time-integrated rate and angular asymmetries, for the exclusive decays $B \rightarrow K^{(*)} \ell^{+} \ell^{-}$[31-37]. The present analysis uses the $424.2 \pm 1.8 \mathrm{fb}^{-1} e^{+} e^{-} \rightarrow \Upsilon(4 S)$ data sample [38], corresponding to $\sim 471 \times 10^{6} B \bar{B}$ pairs, collected with the $B A B A R$ detector $[39,40]$ at the PEP-II collider at the SLAC National Accelerator Laboratory.

The decays $B \rightarrow X_{s} \ell^{+} \ell^{-}$are reconstructed in 10 separate $X_{s}$ hadronic final states $\left(K^{+}, K^{+} \pi^{0}, K^{+} \pi^{-}, K^{+} \pi^{-} \pi^{0}\right.$, $K^{+} \pi^{-} \pi^{+}, K_{S}^{0}, K_{S}^{0} \pi^{0}, K_{S}^{0} \pi^{+}, K_{S}^{0} \pi^{+} \pi^{0}$, and $K_{S}^{0} \pi^{+} \pi^{-}$) [41], combining these with an $e^{+} e^{-}$or $\mu^{+} \mu^{-}$pair for a total of 20 final states. The selection of charged and neutral particle candidates, as well as the reconstruction of $\pi^{0} \rightarrow \gamma \gamma$ and $K_{S}^{0} \rightarrow \pi^{+} \pi^{-}$, is described in Refs. [31,36]. Based on studies including up to $18 X_{s}$ modes with a maximum of four pions and $m_{X_{s}}$ as large as $2.2 \mathrm{GeV} / c^{2}$, we limit the number of $X_{s}$ final states to the 10 listed above and require $m_{X_{s}}<$ $1.8 \mathrm{GeV} / c^{2}$ since the expected signal-to-background ratio rapidly decreases with increasing $X_{s}$ pion multiplicity and mass. We assume that the fraction of modes containing a $K_{L}^{0}$ is equal to that containing a $K_{S}^{0}$ and account for these decays, as well as $K_{S}^{0} \rightarrow \pi^{0} \pi^{0}$ and $\pi^{0}$ Dalitz decays, in our reconstruction efficiencies. With these efficiencies taken into account, the reconstructed states represent $\sim 70 \%$ of the total inclusive rate.

We account for missing hadronic final states, as well as for states with $m_{X_{s}}>1.8 \mathrm{GeV} / c^{2}$, based on the formalism of Refs. [8,13,22,42-44], with hadronization of the $X_{s}$ system provided by the JETSET [45] event generator. Given that we observe no statistically significant nonresonant $B \rightarrow K \pi \ell^{+} \ell^{-}$decays in our data [31], signal decays with a two-body $X_{s}$ system and $m_{X_{s}}<1.1 \mathrm{GeV} / c^{2}$ are assumed

TABLE I. $\quad B \rightarrow X_{s} e^{+} e^{-}, B \rightarrow X_{s} \mu^{+} \mu^{-}$and $B \rightarrow X_{s} \ell^{+} \ell^{-}$partial BFs (in units of $\left.10^{-6}\right)$ and $A_{C P}$ by $q^{2}\left(\mathrm{GeV}^{2} / c^{4}\right)$ and $m_{X_{s}}\left(\mathrm{GeV} / c^{2}\right)$ bin. The number in parentheses after each result is the multiplier which is applied to the measured semi-inclusive rate to account for unreconstructed and $m_{X_{s}}>1.8 \mathrm{GeV} / c^{2}$ final states. Estimated contributions from the vetoed charmonium $q^{2}$ regions are included in both the total and $m_{X_{s}}$ binned results, but not in the total $A_{C P}$. The first uncertainties are statistical, the second experimental systematics and the third model-dependent systematics associated with the multiplicative factor. There are no model-dependent $A_{C P}$ systematics and $A_{C P}$ is not measured as a function of $m_{X_{s}}$; the multiplicative factors are not used in calculating the total $A_{C P}$.

\begin{tabular}{lccccc}
\hline \hline Bin & Range & $B \rightarrow X_{s} e^{+} e^{-}$ & $B \rightarrow X_{s} \mu^{+} \mu^{-}$ & $B \rightarrow X_{s} \ell^{+} \ell^{-}$ & $A_{C P B \rightarrow X_{s} \ell^{+} \ell^{-}}$ \\
\hline$q_{0}^{2}$ & $1.0<q^{2}<6.0$ & $1.93_{-0.45-0.16}^{+0.47+0.21} \pm 0.18(1.71)$ & $0.66_{-0.76-0.24}^{+0.82+0.30} \pm 0.07(1.78)$ & $1.60_{-0.39-0.13}^{+0.41+0.17} \pm 0.18$ & $-0.06 \pm 0.22 \pm 0.01$ \\
$q_{1}^{2}$ & $0.1<q^{2}<2.0$ & $3.05_{-0.49-0.21}^{+0.52+0.29} \pm 0.35(1.96)$ & $1.83_{-0.80-0.24}^{+0.90+0.30} \pm 0.20(2.02)$ & $2.70_{-0.42-0.16}^{+0.45+0.21} \pm 0.35$ & $-0.13 \pm 0.18 \pm 0.01$ \\
$q_{2}^{2}$ & $2.0<q^{2}<4.3$ & $0.69_{-0.28-0.07}^{+0.31+0.11} \pm 0.07(1.73)$ & $-0.15_{-0.43-0.14}^{+0.50+0.26} \pm 0.01(1.80)$ & $0.46_{-0.23-0.06}^{+0.26+0.10} \pm 0.07$ & $0.42_{-0.42}^{+0.50} \pm 0.01$ \\
$q_{3}^{2}$ & $4.3<q^{2}<6.8$ & $0.69_{-0.29-0.10}^{+0.31+0.13} \pm 0.05(1.53)$ & $0.34_{-0.50-0.15}^{+0.54+0.19} \pm 0.03(1.59)$ & $0.60_{-0.25-0.08}^{+0.27+0.10} \pm 0.05$ & $-0.45_{-0.57}^{+0.44} \pm 0.01$ \\
$q_{4}^{2}$ & $10.1<q^{2}<12.9$ & $1.14_{-0.40-0.10}^{+0.42+0.22} \pm 0.04(1.16)$ & $0.87_{-0.47-0.08}^{+0.51+0.11} \pm 0.03(1.18)$ & $1.02_{-0.30-0.07}^{+0.32+0.10} \pm 0.04$ & \\
$q_{5}^{2}$ & $14.2<q^{2}$ & $0.56_{-0.18-0.03}^{+0.19+0.03} \pm 0.00(1.02)$ & $0.60_{-0.29-0.04}^{+0.31+0.05} \pm 0.00(1.02)$ & $0.57_{-0.15-0.02}^{+0.16+0.03} \pm 0.00$ & $\ldots$ \\
$q_{45}^{2}$ & $q_{4}^{2} \cup q_{5}^{2}$ & $\ldots$ & $\ldots$ & $\cdots$ & $\cdots$ \\
$m_{X_{s}, 1}$ & $0.4<m_{X_{s}}<0.6$ & $0.69_{-0.17-0.03}^{+0.18+0.04} \pm 0.00(1.00)$ & $0.74_{-0.23-0.04}^{+0.25+0.04} \pm 0.00(1.00)$ & $0.71_{-0.14-0.03}^{+0.15+0.03} \pm 0.00$ & $0.19_{-0.17}^{+0.18} \pm 0.01$ \\
$m_{X_{s}, 2}$ & $0.6<m_{X_{s}}<1.0$ & $1.20_{-0.33-0.07}^{+0.34+0.10} \pm 0.00(1.00)$ & $0.76_{-0.40-0.07}^{+0.44+0.08} \pm 0.00(1.00)$ & $1.02_{-0.25-0.05}^{+0.27+0.06} \pm 0.00$ & \\
$m_{X_{s}, 3}$ & $1.0<m_{X_{s}}<1.4$ & $1.60_{-0.69-0.19}^{+0.72+0.27} \pm 0.05(1.18)$ & $0.65_{-1.08-0.25}^{+1.16+0.27} \pm 0.02(1.18)$ & $1.32_{-0.58-0.15}^{+0.61+0.19} \pm 0.05$ & \\
$m_{X_{s}, 4}$ & $1.4<m_{X_{s}}<1.8$ & $1.88_{-0.73-0.47}^{+0.76+0.71} \pm 0.12(1.91)$ & $0.19_{-1.25-0.50}^{+1.35+0.70} \pm 0.10(1.91)$ & $1.36_{-0.63-0.54}^{+0.67+0.50} \pm 0.12$ & \\
Total & $0.1<q^{2}$ & $7.69_{-0.77-0.33}^{+0.82+0.50} \pm 0.50$ & $4.41_{-1.17-0.42}^{+1.31+0.57} \pm 0.27$ & $6.73_{-0.64-0.25}^{+0.70+0.34} \pm 0.50$ & $0.04 \pm 0.11 \pm 0.01$ \\
\hline \hline
\end{tabular}


to proceed through the $K^{*}(892)$ resonance. The simulation of such events, as well as those with a single kaon and no pions, is similar to that for inclusive events but incorporates the form factor models of Refs. [46,47].

The kinematic variables $m_{\mathrm{ES}}=\sqrt{E_{\mathrm{c} . \mathrm{m} .}^{2} / 4-p_{B}^{* 2}}$ and $\Delta E=E_{B}^{*}-E_{\text {c.m. }} / 2$, where $p_{B}^{*}$ and $E_{B}^{*}$ are the $B$ momentum and energy in the $\Upsilon(4 S)$ center-of-mass (c.m.) frame with $E_{\mathrm{c} . \mathrm{m}}$. the total c.m. energy, are used to distinguish signal from background events. We require $m_{\mathrm{ES}}>5.225 \mathrm{GeV} / c^{2}$ and $-0.10<\Delta E<0.05 \mathrm{GeV}$ $(-0.05<\Delta E<0.05 \mathrm{GeV})$ for dielectron (dimuon) final states. Signal-like $B$ backgrounds with $J / \psi[\psi(2 S)]$ daughters are removed by vetoing events with $6.8<q^{2}<10.1 \mathrm{GeV}^{2} / c^{4}\left(12.9<q^{2}<14.2\right)$. We reconstruct $X_{s} h^{ \pm} \mu^{\mp}$ final states, where $h$ is a track with no particle identification (PID) requirement applied, to characterize backgrounds from hadrons misidentified as muons. Such backgrounds occur only in dimuon final states because of the significantly higher probability to misidentify $K^{+}$or $\pi^{+}$as a muon rather than an electron. Similarly, backgrounds from $B \rightarrow D\left(\rightarrow K^{(*)} \pi\right) \pi$ decays occur only in dimuon modes and, assigning the pion mass hypothesis to both muon candidates, we reject candidates with $K^{(*)} \pi$ mass values in the range $1.84<m_{K^{(*)} \pi}<2.04 \mathrm{GeV} / c^{2}$.

We suppress $e^{+} e^{-} \rightarrow q \bar{q}$ events (where $q$ is a $u, d, s$, or $c$ quark) and $B \bar{B}$ combinatoric backgrounds using boosted decision trees (BDTs) [48,49] identical in construction to those used in our $B \rightarrow K^{(*)} \ell^{+} \ell^{-}$analysis [31]. These BDTs are, respectively, trained with simulated $u d s c$ or $B \bar{B}$ backgrounds and correctly reconstructed signal events. Ensembles of simulated event samples are used to simultaneously optimize the $\Delta E$ windows and selection on the $u d s c$ BDTs for each individual $q^{2}$ and $m_{X_{s}}$ bin. After all selection criteria are applied, the average multiplicity of $B$ candidates per event is $\sim 2.6(\sim 2.2)$ for $e^{+} e^{-}\left(\mu^{+} \mu^{-}\right)$final states. We allow only one candidate per event, selecting the candidate with the smallest $|\Delta E|$. Signal efficiencies after event selection range from about $1 \%$ to $30 \%$ depending on mode and the $q^{2}$ or $m_{X_{s}}$ bin.

In each $q^{2}$ and $m_{X_{s}}$ bin, we extract the signal yield with a two-dimensional maximum likelihood fit using $m_{\mathrm{ES}}$ and a likelihood ratio $L_{R}$ based on the $B \bar{B}$ BDT, $L_{R} \equiv \mathcal{P}_{\mathrm{S}} /\left(\mathcal{P}_{\mathrm{S}}+\mathcal{P}_{\mathrm{B}}\right)$, where $\mathcal{P}_{\mathrm{S}}$ and $\mathcal{P}_{\mathrm{B}}$ are, respectively, probabilities for genuine-signal and $B \bar{B}$ backgrounds. For correctly reconstructed signal events, $L_{R}$ sharply peaks near one, while $B \bar{B}$ backgrounds peak at zero. Events with $L_{R}>0.42$ are selected. This selection rejects $\gtrsim 95 \%$ of the $B \bar{B}$ background events remaining after all other event selections have been applied, with only a trivial reduction in signal efficiency.

Five (six) event classes contribute to the dielectron (dimuon) maximum likelihood fit: (1) correctly reconstructed signal; (2) events that contain a partially or incorrectly reconstructed $B \rightarrow X_{s} \ell^{+} \ell^{-}$decay (signal cross feed); (3) $u d s c$ and (4) $B \bar{B}$ combinatorial backgrounds;
(5) charmonium backgrounds; and, for dimuon modes, (6) events with hadrons misidentified as muons.

There is no correlation between $m_{\mathrm{ES}}$ and $L_{R}$ for correctly reconstructed signal events. Therefore, the probability distribution function (PDF) for these events is chosen as a product of two 1D PDFs, with $m_{\mathrm{ES}}$ parametrized with a Crystal Ball function [50-52] and $L_{R}$ described by a nonparametric histogram PDF. The Crystal Ball shape parameters are fixed using simulated signal events, as is the $L_{R}$ PDF. These PDFs describe well the $m_{\mathrm{ES}}$ and $L_{R}$ distributions derived from the high-statistics control samples of vetoed signal-like charmonium events. The signal cross feed is modeled as a $2 \mathrm{D} m_{\mathrm{ES}}$ versus $L_{R}$ histogram PDF using simulated signal samples, with normalization $N_{\text {xfd }}$ scaled as a fixed fraction of the fit signal yield $N_{\text {sig. }}$.

The $u d s c$ combinatoric background PDF is derived from simulated events using a 2D nonparametric kernel density estimator with adaptive bandwidth [49,53,54], which is validated using data collected with $e^{+} e^{-}$center-of-mass energy $40 \mathrm{MeV}$ below the $\Upsilon(4 S)$ resonance. The $u d s c$ normalization $N_{u d s c}$ is obtained by scaling the $43.9 \pm$ $0.2 \mathrm{fb}^{-1}$ of off-resonance data [38] by the ratio of on- to off-resonance integrated luminosity.

The shape of the 2D PDF for the $B \bar{B}$ combinatoric background is modeled similarly to the $u d s c$ background. Its normalization in the $5.225<m_{\mathrm{ES}}<5.270 \mathrm{GeV} / c^{2}$ sideband, where no correctly reconstructed signal events are expected, is obtained by subtracting the $N_{\text {xfd }}^{\mathrm{SB}}, N_{\text {udsc }}^{\mathrm{SB}}$, $N_{\mathrm{chm}}^{\mathrm{SB}}$, and $N_{\mathrm{had}}^{\mathrm{SB}}$ (for dimuon events) contributions from the total number of sideband events, giving the $B \bar{B}$ yield in the sideband region $N_{B \bar{B}}^{\mathrm{SB}}$. We use simulated events to obtain the ratio of the number of $B \bar{B}$ combinatoric events in the $m_{\mathrm{ES}}>5.27 \mathrm{GeV} / c^{2}$ signal region to the number in the sideband region to scale $N_{B \bar{B}}^{\mathrm{SB}}$ into the expected contribution $N_{B \bar{B}}$ in the full fit region.

Charmonium backgrounds escaping the vetoed $q^{2}$ regions are similarly described by a 2D kernel estimator, with normalization $N_{\text {chm }}$ derived from a fit to the data in the vetoed regions that is extrapolated into the nonvetoed regions. The normalization $N_{\text {had }}$ and shape of the 2D PDF for misidentified dimuon events are characterized by a weighted 2D histogram taken directly from data using event-by-event weights obtained from PID control samples [31,55].

We extract the $N_{\text {sig }}$ central value and associated upper and lower limits using the negative $\log$-likelihood for $N_{\text {sig. }}$. We calculate partial BFs taking into account the efficiency for each final state in each $q^{2}$ and $m_{X_{s}}$ bin, as well as the multiplicative factors that provide extrapolation to the fully inclusive BFs. The results are shown in Table I, where the fully inclusive total rate and the $m_{X_{\mathrm{s}}}$ binned results include estimated signal contributions in the vetoed charmonium $q^{2}$ regions. Fit projections for all $q^{2}$ and $m_{X_{s}}$ bins are available in the Supplemental Material [56], along with a table giving the raw numerical results from our fits. Figure 1 shows our $q^{2}$ binned results overlaid on the nominal SM expectations 


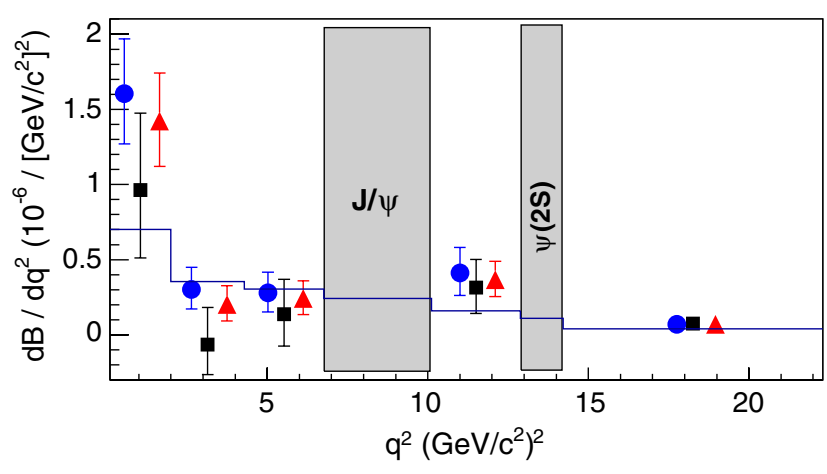

FIG. 1 (color online). Differential BF as a function of $q^{2}$ for electron (blue circles), muon (black squares), and lepton-flavoraveraged final states (red triangles). The errors correspond to the total uncertainties. The histogram shows the SM expectation, which has uncertainties of approximately $10 \%-30 \%$ in different $q^{2}$ regions. The shaded boxes denote the vetoed charmonium regions. The horizontal spread of points in each bin is meant only to aid visibility.

derived from our $B \rightarrow X_{s} \ell^{+} \ell^{-}$signal model. A similar plot for $m_{X_{s}}$ is included in the Supplemental Material [56].

We consider systematic uncertainties associated with purely experimental systematic uncertainties and the model-dependent extrapolation to the fully inclusive rate. The experimental systematics can either be additive, affecting the extraction of the signal yield from the data, or multiplicative, affecting the calculation of a BF from an observed signal yield. Sources of multiplicative systematic uncertainty include $B \bar{B}$ counting as well as tracking, PID, and reconstruction efficiencies. The only significant additive systematic uncertainties are associated with the PDF parametrizations and normalizations. The total experimental systematic uncertainty is the sum in quadrature of the above terms, with the exception that uncertainties related to charged particle tracking efficiencies are assumed to be fully correlated among all charged particles. The evaluation of all experimental systematics is fully described in Ref. [31]. Tables quantifying each individual contribution to the experimental and model-dependent extrapolation systematic uncertainties are available in the Supplemental Material [56].

The uncertainty in the extrapolation to the inclusive rate is characterized through variations that attempt to quantify our lack of knowledge of the true dilepton mass-squared distribution and hadronization of the $X_{s}$ system beyond the specific final states and $m_{X_{s}}$ range that we observe. We average the most recent $B \rightarrow K^{(*)} \ell^{+} \ell^{-}$BFs [57], excluding $B A B A R$ results, and use the latest $B A B A R$ result [58] for the ratio of charged-to-neutral $\Upsilon(4 S) \rightarrow b \bar{b}$ decays, $\Gamma\left(B^{+} B^{-}\right) / \Gamma\left(B^{0} \bar{B}^{0}\right)=1.006 \pm 0.036 \pm 0.031$. Each of these terms is varied by its one-standard-deviation uncertainty. We examine an alternate $m_{X_{s}}$ transition point of $1.0 \mathrm{GeV} / c^{2}$ between the $B \rightarrow K^{(*)} \ell^{+} \ell^{-}$and $B \rightarrow$ $X_{s} \ell^{+} \ell^{-}$models. To account for hadronization uncertainties in $m_{X_{\mathrm{s}}}>1.1 \mathrm{GeV} / c^{2}$ events, we generate 20 simulated data sets with varied JETSET tunings, two different values for the $B$-meson Fermi motion, and two different $b$-quark mass values. We take the full spread of the extrapolation factors derived from these variations to estimate this systematic uncertainty. Additionally, for $m_{X_{\delta}}>1.1 \mathrm{GeV} / c^{2}$, the fraction of modes with more than one $\pi^{0}$ is varied around the generator value of 0.20 by $\pm 50 \%$; the fraction of modes with either no $\pi^{0}$ and more than two charged pions, or one $\pi^{0}$ and more than one charged pion, is varied by $\pm 50 \%$ around the $q^{2}$-dependent generator value; and the fraction of modes with more than one neutral or charged kaon is varied around the generator value of 0.034 by $\pm 50 \%$. Contributions from final states with photons that do not come from $\pi^{0}$ decays but rather from $\eta, \eta^{\prime}, \omega$, etc. are expected to be insignificant, and we do not vary the fractions of these decays. Each of the above variations is added in quadrature to obtain the final model-dependent systematic. Table I lists both the experimental and model-dependent systematics.

We calculate the total inclusive rate by summing the $q_{1}^{2}$ through $q_{5}^{2}$ results taking into account correlations in the systematic uncertainties and estimating signal contributions in the vetoed charmonium $q^{2}$ regions. The lepton-flavoraveraged $B \rightarrow X_{s} \ell^{+} \ell^{-}$results are weighted averages of the individual $B \rightarrow X_{s} e^{+} e^{-}$and $B \rightarrow X_{s} \mu^{+} \mu^{-}$results that take into account correlations in the systematic uncertainties. Figure 1 shows the differential $\mathrm{BF}$ results as a function of $q^{2}$ and $m_{X_{s}}$ overlaid with the SM expectation. The results in these bins, as well as in the $q_{0}^{2}$ region, are generally in good agreement with SM predictions. Given our experimental uncertainties, we are insensitive to the relatively small differences in the $e^{+} e^{-}$and $\mu^{+} \mu^{-}$rates expected in the SM, and observe no significant differences between $e^{+} e^{-}$and $\mu^{+} \mu^{-}$final states.

Several model-independent analyses of the form-factorindependent angular observables reported in a recent $B^{0} \rightarrow K^{+} \pi^{-} \mu^{+} \mu^{-}$LHCb analysis [35] explain the anomaly reported there in terms of a nonvanishing beyond-SM contribution $C_{9}^{\mathrm{BSM}}$ [59-68]. These phenomenological studies all present generally similar results, yielding a $3 \sigma$ range for $C_{9}^{\mathrm{BSM}}$ of $\sim[-2,0]$, implying a corresponding suppression in the fully inclusive $\mathrm{BF}$ of up to $\sim 25 \%$ in the $1<q^{2}<6 \mathrm{GeV}^{2} / c^{4}$ and $q^{2}>14.4 \mathrm{GeV}^{2} / c^{4}$ ranges. Although our results in the $q_{0}^{2}$ range are consistent with both the SM expectation and a possible suppression in the decay rate, our results in the $q_{5}^{2}$ range show an excess, rather than a deficit, of $\sim 2 \sigma$ in both the $B \rightarrow X_{s} e^{+} e^{-}$and $B \rightarrow X_{s} \mu^{+} \mu^{-}$rates with respect to the SM expectation [22].

We search for $C P$ violation in each $q^{2}$ bin by dividing our data set into four disjoint samples according to lepton identity $\left(e^{+} e^{-}\right.$or $\left.\mu^{+} \mu^{-}\right)$and the $B$ or $\bar{B}$ flavor as determined by the kaon and pion charges of the $X_{s}$ system. Modes with $X_{s}=K_{S}^{0}, K_{S}^{0} \pi^{0}$, or $K_{S}^{0} \pi^{+} \pi^{-}$are not used, and, because we perform no model-dependent extrapolation of signal rates, we measure $A_{C P}$ only for the particular combination of final 
states used here. We simultaneously fit all four data sets, sharing a single value of $A_{C P}$ as a free parameter, using the BF's fit model described above. Our $A_{C P}$ results are shown in Table I, and a plot of the results as a function of $q^{2}$ is included as part of the Supplemental Material [56]. We analyze the vetoed $J / \psi$ data set, where $C P$ violation is expected to be trivially small $[69,70]$, with the same fitting methodology used for the signal $q^{2}$ bins; we find $A_{C P}^{c \bar{c} s}=0.0046 \pm 0.0057$ (stat). Observing no significant bias, we assign the statistical uncertainty here as the systematic uncertainty for the $A_{C P}$ results. To extract $A_{C P}$ for the full dilepton mass range, we sum the $A_{C P}$ BFs across the four disjoint $A_{C P} q^{2}$ bins; excluding the charmonium veto windows, we find $A_{C P}=0.04 \pm 0.11$ (stat) \pm 0.01 (syst). We observe no significant asymmetry in any $q^{2}$ region or for the full dilepton mass range.

In summary, we have measured the total and partial BFs, as well as $A_{C P}$, for the inclusive radiative electroweak process $B \rightarrow X_{s} \ell^{+} \ell^{-}$. Our results are in general agreement with SM expectations with the exception of our partial BF results in the high- $q^{2}$ region, which show a $\sim 2 \sigma$ excess compared to both the SM expectation and the most favored value of the beyond-SM contribution $C_{9}^{\mathrm{BSM}}$ advanced to explain recent observations by $\mathrm{LHCb}$ [35].

We are grateful to Enrico Lunghi, Tobias Hurth, and Tobias Huber for useful discussions, as well as providing dilepton mass-squared theory distributions derived using the most up-to-date corrections. We are additionally grateful for the excellent luminosity and machine conditions provided by our PEP-II 2 colleagues, and for the substantial dedicated effort from the computing organizations that support BABAR. The collaborating institutions wish to thank SLAC for its support and kind hospitality. This work is supported by DOE and NSF (U.S.), NSERC (Canada), CEA and CNRS-IN2P3 (France), BMBF and DFG (Germany), INFN (Italy), FOM (Netherlands), NFR (Norway), MES (Russia), MINECO (Spain), and STFC (United Kingdom). Individuals have received support from the Marie Curie EIF (European Union) and the A. P. Sloan Foundation (U.S.).

\footnotetext{
*Deceased.

${ }^{\dagger}$ Now at: University of Tabuk, Tabuk 71491, Saudi Arabia. *Also at: Università di Perugia, Dipartimento di Fisica, I06123 Perugia, Italy.

${ }^{\S}$ Now at: Laboratoire de Physique Nucléaire et de Hautes Energies, IN2P3/CNRS, F-75252 Paris, France.

"Now at: University of Huddersfield, Huddersfield HD1 3DH, United Kingdom.

"Now at: University of South Alabama, Mobile, Alabama 36688, USA.

** Also at: Università di Sassari, I-07100 Sassari, Italy.

${ }^{\dagger}$ Also at: INFN Sezione di Roma, I-00185 Roma, Italy.

*Now at: Universidad Técnica Federico Santa Maria, 2390123 Valparaiso, Chile.
}

[1] G. Buchalla, A.J. Buras, and M.E. Lautenbacher, Rev. Mod. Phys. 68, 1125 (1996).

[2] W. Altmannshofer, P. Paradisi, and D. M. Straub, J. High Energy Phys. 04 (2012) 008.

[3] N. Kosnik, Phys. Rev. D 86, 055004 (2012).

[4] J. Drobnak, S. Fajfer, and J. F. Kamenik, Nucl. Phys. B855, 82 (2012).

[5] S. Descotes-Genon, D. Ghosh, J. Matias, and M. Ramon, J. High Energy Phys. 06 (2011) 099.

[6] S. Oh and J. Tandean, Phys. Rev. D 83, 095006 (2011).

[7] K. S. M. Lee and F. J. Tackmann, Phys. Rev. D 79, 114021 (2009).

[8] A. Ali, E. Lunghi, C. Greub, and G. Hiller, Phys. Rev. D 66, 034002 (2002).

[9] P. Gambino, U. Haisch, and M. Misiak, Phys. Rev. Lett. 94, 061803 (2005).

[10] A. K. Alok, A. Dighe, and S. Ray, Phys. Rev. D 79, 034017 (2009).

[11] A. Soni, A. K. Alok, A. Giri, R. Mohanta, and S. Nandi, Phys. Rev. D 82, 033009 (2010).

[12] J. Beringer et al. (Particle Data Group), Phys. Rev. D 86, 010001 (2012).

[13] H. H. Asatryan, H. M. Asatrian, C. Greub, and M. Walker, Phys. Rev. D 65, 074004 (2002).

[14] H. H. Asatryan, H. M. Asatrian, C. Greub, and M. Walker, Phys. Rev. D 66, 034009 (2002).

[15] A. Ghinculov, T. Hurth, G. Isidori, and Y. P. Yao, Nucl. Phys. B648, 254 (2003).

[16] H. M. Asatrian, K. Bieri, C. Greub, and A. Hovhannisyan, Phys. Rev. D 66, 094013 (2002).

[17] P. Gambino, M. Gorbahn, and U. Haisch, Nucl. Phys. B673, 238 (2003).

[18] A. Ghinculov, T. Hurth, G. Isidori, and Y. P. Yao, Eur. Phys. J. C 33, S288 (2004).

[19] C. Bobeth, P. Gambino, M. Gorbahn, and U. Haisch, J. High Energy Phys. 04 (2004) 071.

[20] A. Ghinculov, T. Hurth, G. Isidori, and Y. P. Yao, Nucl. Phys. B685, 351 (2004).

[21] C. Greub, V. Pilipp, and C. Schupbach, J. High Energy Phys. 12 (2008) 040.

[22] T. Huber, T. Hurth, and E. Lunghi, Nucl. Phys. B802, 40 (2008).

[23] T. Huber, E. Lunghi, M. Misiak, and D. Wyler, Nucl. Phys. B740, 105 (2006).

[24] M. Beneke, G. Buchalla, M. Neubert, and C. T. Sachrajda, Eur. Phys. J. C 61, 439 (2009).

[25] D. S. Du and M. Z. Yang, Phys. Rev. D 54, 882 (1996).

[26] A. Ali and G. Hiller, Eur. Phys. J. C 8, 619 (1999).

[27] C. Bobeth, G. Hiller, and G. Piranishvili, J. High Energy Phys. 07 (2008) 106.

[28] W. Altmannshofer et al., J. High Energy Phys. 01 (2009) 019.

[29] B. Aubert et al. (BABAR Collaboration), Phys. Rev. Lett. 93, 081802 (2004).

[30] M. Iwasaki et al. (Belle Collaboration), Phys. Rev. D 72, 092005 (2005).

[31] J. P. Lees et al. (BABAR Collaboration), Phys. Rev. D 86, 032012 (2012).

[32] J.-T. Wei et al. (Belle Collaboration), Phys. Rev. Lett. 103, 171801 (2009). 
[33] T. Aaltonen et al. (CDF Collaboration), Phys. Rev. Lett. 107, 201802 (2011).

[34] R. Aaij et al. (LHCb Collaboration), Phys. Rev. Lett. 108, 181806 (2012).

[35] R. Aaij et al. (LHCb Collaboration), Phys. Rev. Lett. 111, 191801 (2013).

[36] B. Aubert et al. (BABAR Collaboration), Phys. Rev. D 79, 031102 (2009).

[37] R. Aaij et al. (LHCb Collaboration), Phys. Rev. Lett. 110, 031801 (2013).

[38] J. P. Lees et al. (BABAR Collaboration), Nucl. Instrum. Methods Phys. Res., Sect. A 726, 203 (2013).

[39] B. Aubert et al. (BABAR Collaboration), Nucl. Instrum. Methods Phys. Res., Sect. A 479, 1 (2002).

[40] B. Aubert et al. (BABAR Collaboration), Nucl. Instrum. Methods Phys. Res., Sect. A 729, 615 (2013).

[41] The use of charge conjugate reactions is implied unless otherwise indicated.

[42] F. Kruger and L. M. Sehgal, Phys. Lett. B 380, 199 (1996).

[43] A. Ali, G. Hiller, L. T. Handoko, and T. Morozumi, Phys. Rev. D 55, 4105 (1997).

[44] C. Bobeth, M. Misiak, and J. Urban, Nucl. Phys. B574, 291 (2000).

[45] T. Sjöstrand, Comput. Phys. Commun. 82, 74 (1994).

[46] P. Ball and R. Zwicky, Phys. Rev. D 71, 014029 (2005).

[47] P. Ball and R. Zwicky, Phys. Rev. D 71, 014015 (2005).

[48] L. Breiman, Mach. Learn. 24, 123 (1996).

[49] I. Narsky, arXiv:physics/0507157.

[50] M. J. Oreglia, Ph.D. thesis, Stanford University (Report No. SLAC-236, 1980).

[51] J. E. Gaiser, Ph.D. thesis, Stanford University (Report No. SLAC-255, 1982).

[52] T. Skwarnicki, Report No. DESY-F31-86-02.

[53] E. Parzen, Ann. Math. Stat. 33, 1065 (1962).

[54] V. A. Epanechnikov, Theory Probab. Appl. 14, 153 (1969).
[55] B. Aubert et al. (BABAR Collaboration), Phys. Rev. D 73, 092001 (2006).

[56] See Supplemental Material at http://link.aps.org/ supplemental/10.1103/PhysRevLett.112.211802 for plots of our results as a function of $q^{2}$ and $m x$, as well as tables of systematics and raw fitted numerical yields, and projections of each of our branching fraction fits onto their respective data sets.

[57] Y. Amhis et al. (Heavy Flavor Averaging Group), arXiv:1207.1158.

[58] B. Aubert et al. (BABAR Collaboration), Phys. Rev. D 69, 071101 (2004).

[59] S. Descotes-Genon, J. Matias, and J. Virto, Phys. Rev. D 88, 074002 (2013).

[60] S. Jäger and J. Martin Camalich, J. High Energy Phys. 05 (2013) 043.

[61] A. J. Buras and J. Girrbach, J. High Energy Phys. 12 (2013) 009.

[62] W. Altmannshofer and D. M. Straub, Eur. Phys. J. C 73, 2646 (2013).

[63] R. Gauld, F. Goertz, and U. Haisch, Phys. Rev. D 89, 015005 (2014).

[64] R. Gauld, F. Goertz, and U. Haisch, J. High Energy Phys. 01 (2014) 069.

[65] F. Beaujean, C. Bobeth, and D. van Dyk, arXiv:1310.2478.

[66] R. R. Horgan, Z. Liu, S. Meinel, and M. Wingate, arXiv:1310.3887 [Phys. Rev. Lett. (to be published)].

[67] A. J. Buras, F. De Fazio, and J. Girrbach, J. High Energy Phys. 02 (2014) 112.

[68] T. Hurth and F. Mahmoudi, J. High Energy Phys. 04 (2014) 097.

[69] G.-H. Wu and A. Soni, Phys. Rev. D 62, 056005 (2000).

[70] W.-S. Hou, M. Nagashima, and A. Soddu, arXiv:hep-ph/ 0605080 . 